\title{
The Relationship between Thermal Tolerance of Cereal Aphids and their Bacterial Symbionts ${ }^{\dagger}$
}

\author{
Muhammad Zeeshan Majeed ${ }^{1,2,}$ * Chun-Sen Ma ${ }^{1}$, Ahmed Raza ${ }^{3,4}$ and Zhang Bo ${ }^{1}$ \\ 1 Climate Change Biology Research Group, State Key Laboratory for Biology of Plant Diseases and Insect \\ Pests, Institute of Plant Protection, Chinese Academy of Agricultural Sciences, Beijing 100193, PR China; \\ machunsen@caas.cn (C.-S.M.); zhangbo05@caas.cn (Z.B.) \\ 2 Department of Entomology, College of Agriculture, University of Sargodha, 40100 Sargodha, Pakistan \\ 3 Cereal Fungal Diseases Research Group, State Key Laboratory for Biology of Plant Diseases and Insect \\ Pests, Institute of Plant Protection, Chinese Academy of Agricultural Sciences, Beijing 100193, PR China; \\ ahmed_scuafdo@yahoo.com \\ 4 Department of Plant Pathology, University of Agriculture, Sub-Campus Depalpur, 56300 Okara, Pakistan \\ * Correspondence: zeeshan.majeed@uos.edu.pk \\ + Presented at the 1st International Electronic Conference on Entomology (IECE 2021), 1-15 July 2021; \\ Available online: https://iece.sciforum.net/.
}

Citation: Majeed, M.Z.; Ma, C.-S., F.; Raza, A.; Zhang, B. The Relationship between Thermal Tolerance of Cereal Aphids and their Bacterial Symbionts, in Proceedings of the 1st International Electronic Conference on Entomology, 1-15 July 2021, MDPI: Basel, Switzerland,

doi:10.3390/IECE-10521

Published: 1 July 2021

Publisher's Note: MDPI stays neutral with regard to jurisdictional claims in published maps and institutional affiliations.

Copyright: () 2021 by the authors. Submitted for possible open access publication under the terms and conditions of the Creative Commons Attribution (CC BY) license (http://creativecommons.org/licenses/by/4.0/).

\begin{abstract}
This study was aimed to determine if there was any effect of chronic heat exposures and heat acclimation on the thermal thresholds and on the abundance of symbiotic bacteria of cereal aphids Rhopalosiphum padi (L.) and Sitobion avenae (F.). Aphid clones were randomly collected from the wheat fields and were reared in laboratory under controlled conditions. Thermal tolerance indices (chronic, basal and acclimated CTmax) were determined for five-day old apterous female aphids. Real time quantitative-PCR was used to assess the total eubacterial (16S) and aphid-specific bacterial symbiont gene abundance in aphids. Averagely, $R$. padi were more tolerant to chronic heat exposure (to $31^{\circ} \mathrm{C}$ ) and its CTmax values were $1.0^{\circ} \mathrm{C}$ higher than $S$. avenae. Aphid-specific symbiont genes abundance per aphid was almost similar for both of the species. Moreover, for both species, temperature-tolerant aphids exhibited significantly higher symbiont genes than the susceptible aphids. Likewise, thermal tolerance of both aphid species were found correlated with the gene abundance of total symbionts (16S), Buchnera aphidicola, Serratia symbiotica, Hamiltonella defensa, Regiella insecticola, Rickettsia spp. and Spiroplasma spp., suggesting their potential role in conferring thermal tolerance to these aphids.
\end{abstract}

Keywords: Bird cherry-oat aphid; English grain aphid; Thermal threshold; CTmax; Acclimation; bacterial symbionts; Buchnera aphidicola; Serratia symbiotica

\section{Introduction}

Temperature change has been a focal point while demonstrating the direct and indirect effects of climate change on terrestrial organisms including herbivorous insect pests [1-4]. Many studies have demonstrated the pronounced effects of chronic and acclimated exposures to high temperatures on the thermal biology and physiology of insects including aphids [5-9]. As aphids harbor a dense and diverse community of intra- and extracellular microbial symbionts performing various obligatory and facultative functions [1012 ] and this sap-feeding insects have been a model system for investigating insect-microbial symbionts interactions [13-16], the potential impact of thermal exposures (either chronic or acclimated) on the abundance of their gut microbial symbionts has not been elucidated yet.

This study aimed to understand how extreme temperature events would alter the thermal tolerance and symbiotic bacterial abundance of cereal aphids Rhopalosiphum padi (Linnaeus) and Sitobion avenae (Fabricius). Secondary objective was to find out if there is 
any correlation among the thermal tolerance traits of aphids and their respective symbiotic bacteria?

\section{Methods}

\subsection{Insect Collection and Culture}

Two cereal aphids, i.e. bird cherry-oat aphid R. padi and English grain aphid S. avenae, were used as model species in this study due to their differential behaviour and population performance to extreme temperature regimes [9,17]. Ninety nine wild clones of each aphid species were collected randomly from different winter wheat (Triticum aestivum L.) fields near Hebei and Henan provinces of China and were reared separately up to $\mathrm{F}_{3}$ generation under controlled temperature conditions at $20 \pm 2^{\circ} \mathrm{C}, 65 \pm 5 \% \mathrm{RH}$ and $16 \mathrm{~h} \mathrm{~L}: 8 \mathrm{~h} \mathrm{D}$ photoperiod.

\subsection{Thermal Exposure Experiments}

Three batches were made from each of the laboratory reared aphid generation. Each batch was composed of 33 five-day old healthy and active apterous adults. One batch was exposed chronically to a temperature of $31^{\circ} \mathrm{C}$ till death in an environment chamber and aphid mortality was recorded at regular intervals of 3-6 h. We divided and preserved the aphid individuals into four batches according to their tolerance to chronic temperature $\left(31^{\circ} \mathrm{C}\right)$, One batch was used for the determination of basal critical thermal maxima (CTmax) till death, while the other batch of 33 aphids was first acclimated for $3 \mathrm{~h}$ to a constant temperature of $34^{\circ} \mathrm{C}$ prior to the determination of CTmax which was determined using a programmed glycol bath. For determining acclimation temperature, preliminary study showed that aphids can be acclimated up to $3 \mathrm{~h}$ without losing their fitness. After CTmax experiment, dead aphid individuals were immediately preserved in $95 \%$ ethanol at $-20^{\circ} \mathrm{C}$ for DNA extraction.

\subsection{Quantification of Aphid Symbionts Gene Copy Numbers}

Using TIANamp ${ }^{\circledR}$ genomic DNA Kit (Tiangen Biotech, China), total DNA from the preserved aphid individuals was extracted according to manufacturer's protocol. Diagnostic PCR assays were performed for detection and optimization of annealing temperatures of primers using thermal cycler (Bio-Rad). For qPCR, linearized recombinant plasmids were prepared and standard curves were determined from 10-fold serial dilutions of the linearized plasmids containing $10^{1}$ to $10^{9}$ copies of targeted bacterial genes. Three independent technical and biological replicates were determined for each sample.

\subsection{Statistical Analysis}

Statistical analysis of data was done using analytical software Statistix V. 8.1 ${ }^{\circledR}$. Data values were compared using one-way analysis of variance (ANOVA) followed by Fisher's least significant difference (LSD) post-hoc test at standard level of significance $(\alpha=0.05)$. Gene copy numbers of aphid-specific bacterial symbionts among different treatments or aphid species were compared using Student's paired $t$-test at $p \leq 0.05$.

\section{Results}

\subsection{Chronic Thermal Tolerance and Symbionts Gene Abundance}

Upon chronic thermal exposure (to $31^{\circ} \mathrm{C}$ ) of $5 \mathrm{~d}$ old apterous aphid individuals, mortality was less than $5 \%$ within first $36 \mathrm{~h}$ and $18 \mathrm{~h}$ of exposure for $R$. padi and S. avenae, respectively, but then significantly increased and significant portion of the exposed aphid individuals (almost 75\%) died between 36 to $66 \mathrm{~h}$ for $R$. padi and between 18 to $36 \mathrm{~h}$ for $S$. avenae.

Moreover, qPCR results revealed that tolerant (T) and highly tolerant (HT) aphid individuals of $R$. padi exhibited significantly higher copy numbers of total bacterial (16S 
rDNA) and all of the endosymbiotic bacterial genes i.e. B. aphidicola, S. symbiotica, $H$. defensa, R. insecticola and Rickettsia spp. (Figure 2). However, in case of S. avenae, the difference was only significant for $16 S$ rDNA, S. symbiotica and Rickettsia spp. only (Figure 3).

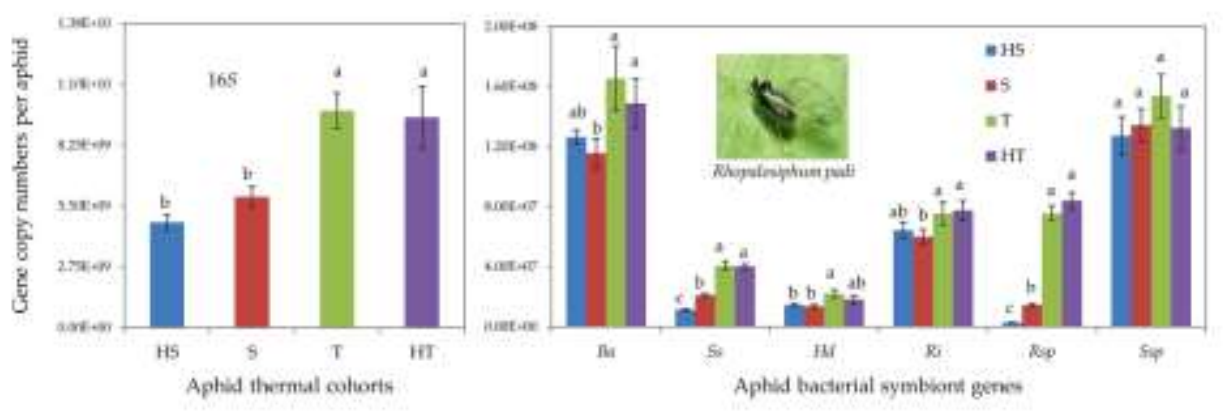

Figure 2. Gene copy numbers (mean $\pm \mathrm{SD}$ ) of aphid-specific bacterial symbionts in different thermal cohorts of Rhopalosiphum padi adults exposed chronically till death to $31^{\circ} \mathrm{C}$. Letters at bar tops indicate significant difference among the treatments (one-way ANOVA; $p \leq 0.05$ ).
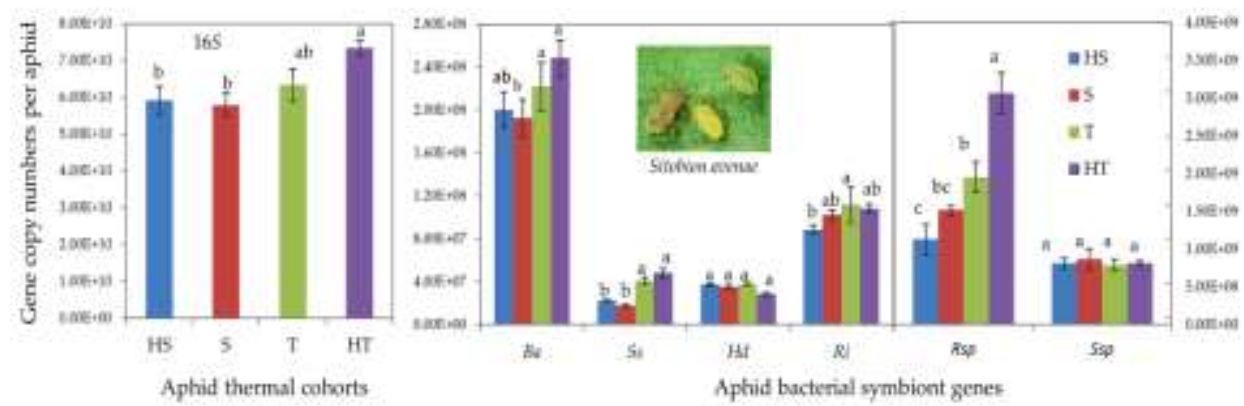

Figure 3. Gene copy numbers (mean $\pm \mathrm{SD}$ ) of aphid-specific bacterial symbionts in different thermal cohorts of Sitobion avenae adults exposed chronically till death to $31^{\circ} \mathrm{C}$. Letters at bar tops indicate significant difference among the treatments (one-way ANOVA; $p \leq 0.05$ ).

\subsection{Critical Thermal Tolerance and Symbionts Gene Abundance}

Average basal and acclimated thermal thresholds (CTmax indices) were $37.41 \pm 0.48$ ${ }^{\circ} \mathrm{C}$ and $38.82 \pm 0.44{ }^{\circ} \mathrm{C}$ for $R$. padi and $36.79 \pm 0.46{ }^{\circ} \mathrm{C}$ and $37.53 \pm 0.51{ }^{\circ} \mathrm{C}$ for S. avenae. On average, $R$. padi thermal threshold values were approximately $1.0^{\circ} \mathrm{C}$ higher than those of $S$. avenae. For both species, acclimated aphid individuals exhibited significantly higher thermal threshold (CTmax) than those of basal treatments $(p<0.001)$. Moreover, thermal thresholds (both basal and acclimated CTmax indices) gradually increased from parental to $\mathrm{F}_{3}$ generation for both aphid species, (data not shown).

In case of R. padi, the abundance of B. aphidicola, S. symbiotica, H. defensa and R. insecticola were significantly higher in acclimated aphid individuals than the non-acclimated (basal) ones (Figure 5). Similarly, gene copy numbers of B. aphidicola, S. symbiotica and $H$. defensa are found slightly but significantly higher in acclimated S. avenae aphid individuals than non-acclimated ones (Figure 6). Thermal tolerance indices were found significantly correlated with the gene abundance (copy numbers) of total symbionts, B. aphidicola, $S$. symbiotica and R. insecticola for R. padi, while with the gene abundance of total symbionts, B. aphidicola, S. symbiotica, Rickettsia spp. and Spiroplasma spp. for S. avenae (data not shown). 

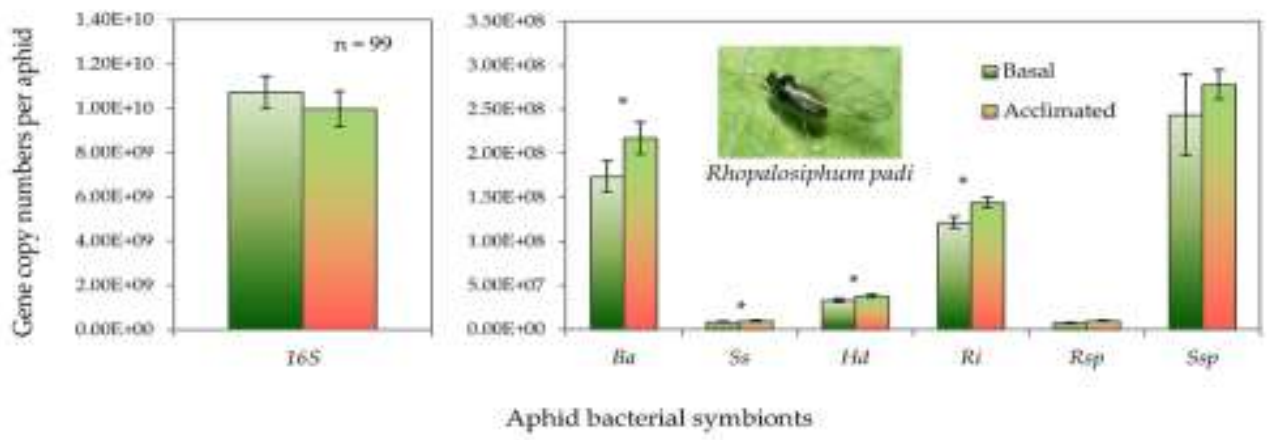

Figure 5. Gene copy numbers (mean $\pm \mathrm{SD}$ ) of aphid-specific bacterial symbionts in basal and acclimated Rhopalosiphum padi adults. Asterisk $\left(^{*}\right)$ symbol indicates significant difference among both basal and acclimated treatments (Student's paired $t$-test; $p \leq 0.05$ ).
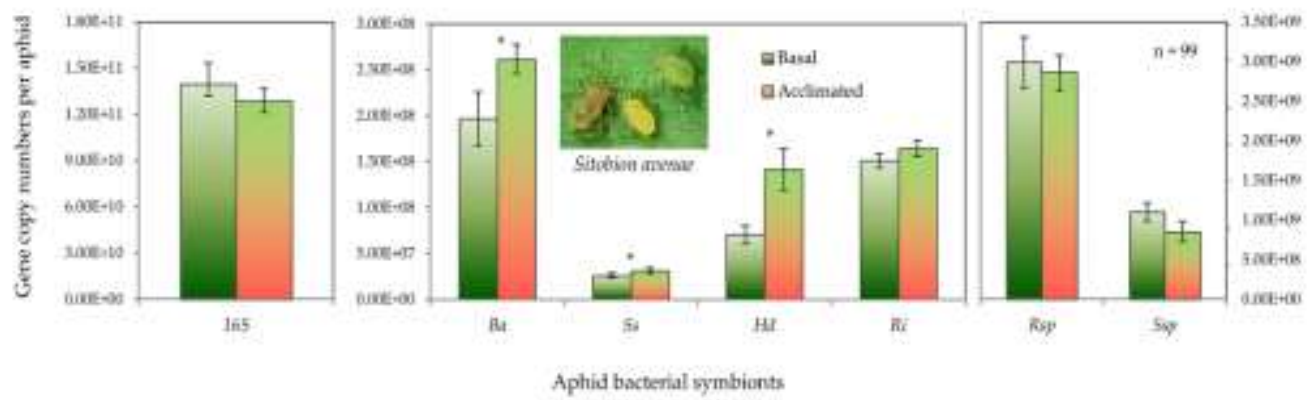

Figure 6. Gene copy numbers (mean \pm SD) of aphid-specific bacterial symbionts in basal and acclimated Sitobion avenae adults. Asterisk $\left({ }^{*}\right)$ symbol indicates significant difference among both basal and acclimated treatments (Student's paired $t$-test; $p \leq 0.05$ ).

\section{Discussion}

This study was aimed to determine the impact of different heat treatments or high temperature exposures on the thermal thresholds of cereal aphids R. padi and S. avenae and on the abundance of their primary (obligate) endosymbiotic bacterium B. aphidicola and secondary (facultative) endosymbiotic bacterial symbionts (i.e. S. symbiotica, $\mathrm{H}$. defensa, R. insecticola, Richettsia spp. and Spiroplasma spp. [16,18].

Results revealed that $R$. padi individuals were more tolerant to chronic thermal exposure than S. avenae and averagely exhibited $1.0^{\circ} \mathrm{C}$ higher thermal threshold (basal and acclimated CTmax) values than those of $S$. avenae. These results regarding the speciesspecific differential heat tolerance and/or thermal traits are consistent with the findings of Zhu et al. [17] who demonstrated that $R$. padi aphid species had higher evolutionary potential when exposed to extreme high-temperature events while $S$. avenae had less fitness under thermal extremes. Our results are in line with previous studies showing that $S$. avenae is more heat sensitive than R. padi $[7,19]$.

For both species, acclimated aphid individuals exhibited significantly higher thermal threshold (CTmax) than those of basal treatments. These results are line with Zhu et al. [17] revealing that acclimated $R$. padi had a higher heat tolerance (CTmax) and fitness than S. avenae. Enhanced thermal threshold and plasticity induced by the acclimation to elevated temperatures has been documented in other organism as well including mites [20] and aquatic holobiont (hydra/algae) systems [21].

Moreover, it was found that as compared to susceptible ones, the cohorts of tolerant aphid individuals of both species harbored significantly higher bacterial symbiont genes particularly of B. aphidicola, S. symbiotica, R. insecticola and Rickettsia spp. Similarly, B. aphidicola, S. symbiotica and $H$. defensa gene abundance were significantly higher in acclimated aphid individuals than non-acclimated (basal) ones. Moreover, thermal tolerance indices correlated significantly with the abundance (gene copy numbers) of total symbionts, $B$. 
aphidicola, S. symbiotica and R. insecticola for R. padi, while with the gene abundance of total symbionts, B. aphidicola, S. symbiotica, Rickettsia spp. and Spiroplasma spp. for S. avenae. These findings substantiate the putative role of these aphid specific bacterial symbionts in mediating host's thermal tolerance [22,23]. Russell and Moran [23] demonstrated that aphid symbionts $S$. symbiotica and $H$. defensa confer tolerance to high temperature exposures. Overall, our results suggest that aphid-bacterial symbiont interactions may play a crucial role in the thermal adaptation of hosts to extreme temperature events or exposures.

\section{Conclusions}

In brief, study revealed that $R$. padi and S. avenae individuals tolerant to chronic thermal exposures harbored significantly higher bacterial symbiont abundance than the susceptible ones. Acclimation to $34{ }^{\circ} \mathrm{C}$ for $3 \mathrm{~h}$ significantly increased the thermal tolerance (CTmax) and bacterial symbiotic gene abundance for both aphid species. On average, $R$. padi thermal threshold values were approximately $1.0^{\circ} \mathrm{C}$ higher than those of $S$. avenae. For both species, the abundance of B. aphidicola, S. symbiotica, H. defensa and R. insecticola were significantly higher in acclimated aphid individuals than basal (non-acclimated ones) and were found positively correlated with the basal and acclimated thermal indices.

Funding: This research work was financially supported by Talented Young Scientist Program (TYSP) sponsored by the Ministry of Science and Technology (MoST) of China.

Institutional Review Board Statement: Not applicable.

Conflicts of Interest: The authors declare no conflict of interest.

\section{References}

1. Bale, J.S.; Masters, G.J.; Hodkinson, I.D.; Awmack, C.; Bezemer, T.M.; Brown, V.K.; Butterfield, J., Buse, A.; Coulson, J.C.; Farrar, J.; Good, J.E. Herbivory in global climate change research: direct effects of rising temperature on insect herbivores. Glob Chang Biol 2002, 8,1-6.

2. Botkin, D.B., Saxe, H., Araujo, M.B., Betts, R., Bradshaw, R.H., Cedhagen, T., Chesson, P., Dawson, T.P., Etterson, J.R., Faith, D.P., Ferrier, S. Forecasting the effects of global warming on biodiversity. Biosci. 2007, 57, 227-36.

3. Bellard, C.; Bertelsmeier, C.; Leadley, P.; Thuiller, W.; Courchamp, F. Impacts of climate change on the future of biodiversity. Ecol. Lett. 2012, 15, 365-77.

4. Colinet, H., Sinclair, B.J., Vernon, P., Renault, D. Insects in fluctuating thermal environments. Ann. Rev. Entomol. 2015, 60, 123140.

5. Hofstetter, R., Dempsey, T., Klepzig, K., Ayres, M. Temperature-dependent effects on mutualistic, antagonistic, and commensalistic interactions among insects, fungi and mites. Comm. Ecol. 2007, 8, 47-56.

6. Musolin, D.L. Insects in a warmer world: ecological, physiological and life-history responses of true bugs (Heteroptera) to climate change. Glob. Chang. Biol. 2007, 13, 1565-1585.

7. Ma, G., Ma, C.S. Effect of acclimation on heat-escape temperatures of two aphid species: implications for estimating behavioral response of insects to climate warming. J. Insect Physiol. 2012, 58, 303-309.

8. Ma, C.S., Ma, G., Pincebourde, S. Survive a warming climate: insect responses to extreme high temperatures. Ann. Rev. Entomol. 2020, 66, 163-184.

9. Ma, G., Rudolf, V.H., Ma, C.S. Extreme temperature events alter demographic rates, relative fitness, and community structure. Glob. Chang. Biol. 2015, 21, 1794-808.

10. Moran, N.A., Plague, G.R., Sandström, J.P., Wilcox, J.L. A genomic perspective on nutrient provisioning by bacterial symbionts of insects. Proc. Nat. Acad. Sci. 2003, 100, 14543-14548.

11. Hansen, A.K., Moran, N.A. The impact of microbial symbionts on host plant utilization by herbivorous insects. Mol. Ecol. 2014, 23, 1473-1496.

12. Sabater-Muñoz, B., Toft, C. Evolution from free-living bacteria to endosymbionts of insects: genomic changes and the importance of the chaperonin GroEL. In Symbiosis: Cellular, Molecular, Medical and Evolutionary Aspects, Kloc, M. Ed.; Springer, Cham. Switzerland, 2020, pp. 77-103.

13. Tsuchida, T., Koga, R, Shibao, H., Matsumoto, T., Fukatsu, T. Diversity and geographic distribution of secondary endosymbiotic bacteria in natural populations of the pea aphid, Acyrthosiphon pisum. Mol. Ecol. 2002, 11, 2123-35.

14. Moran, N.A., Russell, J.A., Koga, R., Fukatsu, T. Evolutionary relationships of three new species of Enterobacteriaceae living as symbionts of aphids and other insects. Appl. Environ. Microbiol. 2005, 71, 3302-3310. 
15. Sakurai, M., Koga, R., Tsuchida, T., Meng, X.Y., Fukatsu, T. Rickettsia symbiont in the pea aphid Acyrthosiphon pisum: novel cellular tropism, effect on host fitness, and interaction with the essential symbiont Buchnera. App. Environ. Microbiol. 2005, 71, 4069-75.

16. Zytynska, S.E., Weisser, W.W. The natural occurrence of secondary bacterial symbionts in aphids. Ecol. Entomol. 2016, 41, 1326.

17. Zhu, L., Hoffmann, A.A., Li, S.M., Ma, C.S. Extreme climate shifts pest dominance hierarchy through thermal evolution and transgenerational plasticity. Func. Ecol. 2021, 1-14. DOI: 10.1111/1365-2435.13774.

18. Sepúlveda, D.A., Zepeda-Paulo, F., Ramírez, C.C., Lavandero, B., Figueroa, C.C. Diversity, frequency and geographic distribution of facultative bacterial endosymbionts in introduced aphid pests. Insect Sci. 2017, 24, 511-521.

19. Turak, E., Talent, R., Sunnucks, P., Hales, D.F. Different responses to temperature in three closely-related sympatric cereal aphids. Entomol. Exp. Appl. 1998, 86, 49-58.

20. Hart, A.J., Bale, J.S., Tullett, A.G., Worland, M.R., Walters, K.F.A. Effects of temperature on the establishment potential of the predatory mite Amblyseius californicus McGregor (Acari: Phytoseiidae) in the UK. J. Insect Physiol. 2002, 48, 593-599.

21. Ye, S., Badhiwala, K.N., Robinson, J.T., Cho, W.H., Siemann, E. Thermal plasticity of a freshwater cnidarian holobiont: detection of trans-generational effects in asexually reproducing hosts and symbionts. ISME J. 2019, 13, 2058-2067.

22. Montllor, C.B., Maxmen, A., Purcell, A.H. Facultative bacterial endosymbionts benefit pea aphids Acyrthosiphon pisum under heat stress. Ecol. Entomol. 2002, 27, 189-195.

23. Russell, J.A., Moran, N.A. Costs and benefits of symbiont infection in aphids: variation among symbionts and across temperatures. Proc. Royal Soc. Biol. Sci. 2006, 273, 603-610. 